\title{
Spatial solitons in nonlinear left-handed metamaterials
}

\author{
Ilya V. Shadrivov and Yuri S. Kivshar \\ Nonlinear Physics Group, Research School of Physical Sciences and Engineering, \\ Australian National University, Canberra ACT 0200, Australia
}

\begin{abstract}
We predict that nonlinear left-handed metamaterials can support both TE- and TM-polarized selftrapped localized beams, spatial electromagnetic solitons. Such solitons appear as single- and multihump beams, being either symmetric or antisymmetric, and they can exist due to the hysteresis-type magnetic nonlinearity and the effective domains of negative magnetic permeability.

PACS numbers: 78.20.Ci, 41.20.Jb, 42.81.Dp
\end{abstract}

Left-handed metamaterials, i.e. materials with simultaneously negative real parts of dielectric permittivity and magnetic permeability [1], demonstrate many peculiar properties including negative refraction and subwavelength imaging. A number of recent experiments reported the observation of some of those properties for artificially fabricated composites [2, 3] and planar transmission-line structures [4] at microwave frequencies. There are strong efforts to fabricate the left-handed composite materials operating at other frequencies, and negative magnetic permeability at $\mathrm{THz}$ frequencies has been recently demonstrated in planar structures composed of nonmagnetic conductive resonant elements [5].

Composite materials used for demonstrating the properties of left-handed media and negative refraction possess combined negative electric and magnetic response in some finite frequency range determined by the resonant properties of nonmagnetic conductive elements of the artificial composites. Nonlinearity introduced in such materials would allow to control the frequency domain with the left-handed properties through strong modification of the effective nonlinear magnetic response [ 6 ]. In particular, both dielectric permittivity and magnetic permeability of the composite material consisting of a mesh of wires and split-ring resonators embedded into a nonlinear dielectric can be controlled by varying the electromagnetic field intensity in such a way that it may allow switching the material properties between left- and righthanded [6]. Since microscopic fields can be enhanced dramatically in a composite structure due to the split-ring resonators, strong nonlinear effects are expected to be important in such types of metamaterials, and this can stimulate applications of nonlinear left-handed materials for switching devices, limiters, and frequency conversion. In addition, different types of nonlinear elements can also be easily introduced into planar left-handed transmissionline structures [4] making a response of such a structure strongly nonlinear.

In this Letter, we study, for the first time to our knowledge, self-trapping of electromagnetic waves and the formation of spatial solitons in nonlinear left-handed composite media, and find the conditions for the existence of both TE- and TM-polarized bright and dark solitons [7]. We demonstrate that left-handed materials with a hysteresis-type (multi-stable) nonlinear magnetic response support novel and unique types of single- and multi-hump (symmetric, antisymmetric, or even asymmetric) backward-wave spatial electromagnetic solitons due to the effective domains corresponding to different branches of nonlinear magnetic permeability.

We consider a nonlinear composite structure described, within an effective medium approximation, by the nonlinear magnetic permeability of the form [ $[$ ]

$$
\mu\left(\omega ;|H|^{2}\right)=1+\frac{F \omega^{2}}{\omega_{0 N L}^{2}\left(|H|^{2}\right)-\omega^{2}+i \Gamma \omega},
$$

where $H$ is the applied magnetic field, $F$ is a filling factor of the composite, $\omega_{0 N L}$ is a nonlinear eigenfrequency of the split-ring resonator, and $\Gamma$ is the loss coefficient. We assume that the infilling dielectric of the structure has a Kerr-like nonlinear response,

$$
\epsilon_{D}\left(|E|^{2}\right)=\epsilon_{D 0}+\alpha|E|^{2} / E_{c}^{2}
$$

where $\epsilon_{D 0}$ is the linear dielectric permittivity, $E_{c}$ is a characteristic electric field of a nonlinear response, and $\alpha= \pm 1$ stands for focussing or defocusing nonlinearity, respectively. Here we neglect the dependence of the effective dielectric permittivity on the electric field assuming that a nonlinear dielectric fills only the slits of the splitring resonators, as discussed in Ref. $[\underline{6}$.

Dependence of the nonlinear eigenfrequency of splitring resonators on the macroscopic magnetic field $H$ can be found in an implicit form (see Eq. (11) of Ref. [6]),

$$
|H|^{2}=\alpha A^{2} \frac{\left(1-X^{2}\right)\left[\left(X^{2}-\Omega^{2}\right)^{2}+\Omega^{2} \gamma^{2}\right]}{X^{6}},
$$

where $X=\omega_{0 N L} / \omega_{0}$ is the normalized nonlinear frequency, $A^{2}=16 \epsilon_{D 0}^{3} \omega_{0}^{2} h^{2} / c^{2}, \Omega=\omega / \omega_{0}, \omega_{0}=$ $(c / a)\left[d_{g} / \pi h \epsilon_{D 0}\right]^{1 / 2}$ is the linear frequency of a split-ring resonator, $\Gamma^{\prime}=\Gamma / \omega_{0}$ is the normalized damping coefficient, $c$ is the speed of light, $d_{g}, h, a$ are the resonator parameters, and the magnetic field $H$ is normalized to the critical electric field $E_{c}$.

When the losses are negligible, the dependence of the magnetic permeability on the normalized magnetic field $H$ can have several distinct forms [ $[$ ] . The most interesting, multi-valued dependence is shown in the inset Fig. [ for the defocusing nonlinearity and $\Omega>1$. 


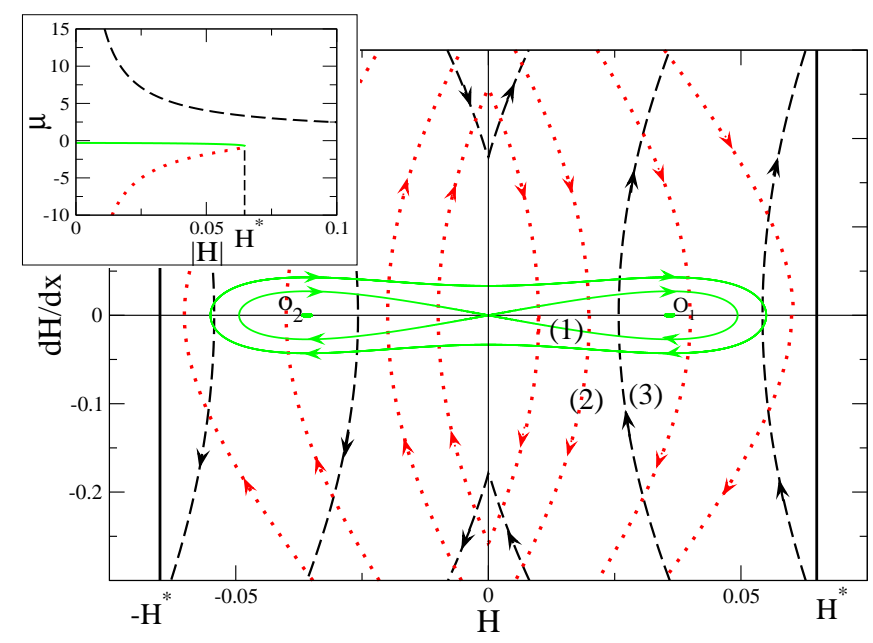

FIG. 1: (color online) Phase trajectories on the plane $(H, d H / d x)$ described by Eq. (4) at $\gamma=2.8$ with the nonlinear magnetic response shown in the inset for $\alpha=-1, \epsilon_{D 0}=12.8$, $h=0.03 \mathrm{~cm}, d_{g}=0.01 \mathrm{~cm}, a=0.3 \mathrm{~cm}$, and $F=0.4$. Solid, dashed, and dotted lines mark the phase trajectories corresponding to the solid, dashed and dotted branches of the nonlinear magnetic permeability.

TM-polarized solitons. First, we study spatially localized TM-polarized waves that are described by one component of the magnetic field and two components of the electric field. We consider monochromatic stationary waves with the magnetic field component $H=H_{y}$ propagating along the $z$-axis and homogeneous in the $y$-direction, $[\sim \exp (i \omega t-i k z)]$, described by the dimensionless nonlinear Helmholtz equation

$$
\frac{d^{2} H}{d x^{2}}+\left[\epsilon \mu\left(|H|^{2}\right)-\gamma^{2}\right] H=0
$$

where $\gamma=k c / \omega$ is a wavenumber, $x=x^{\prime} \omega / c$ is the dimensionless coordinate, and $x^{\prime}$ is the dimensional coordinate. Different types of localized solutions of Eq. (4) can be analyzed on the phase plane $(H, d H / d x)$ (see, e.g., Refs. [8, 9]). First, we find the equilibrium points: the point $(0,0)$ existing for all parameters, and the point $\left(0, H_{1}\right)$, where $H_{1}$ is found from Eq. (3) at

$$
X^{2}\left(H_{1}\right)=X_{\mathrm{eq}}^{2}=\Omega^{2}\left\{1+\frac{F \epsilon_{\mathrm{eff}}}{\left(\gamma^{2}-\epsilon_{\mathrm{eff}}\right)}\right\} .
$$

Below the threshold, i.e. for $\gamma<\gamma_{\mathrm{tr}}$, where $\gamma_{\mathrm{tr}}^{2}=$ $\epsilon\left[1+F \Omega^{2} /\left(1-\Omega^{2}\right)\right]$, the only equilibrium state $(0,0)$ is a saddle point and, therefore, no finite-amplitude or localized waves can exist. Above the threshold value, i.e. for $\gamma>\gamma_{\mathrm{tr}}$, the phase plane has three equilibrium points, as shown in Fig. 1 for the particular value $\gamma=2.8$.

In the vicinity of the equilibrium state $(0,0)$, linear solutions of Eq. (44) describe either exponentially growing or exponentially decaying modes. The equilibrium state $\left(0, H_{1}\right)$ describes a finite-amplitude wave mode of the transverse electromagnetic field. In the region of

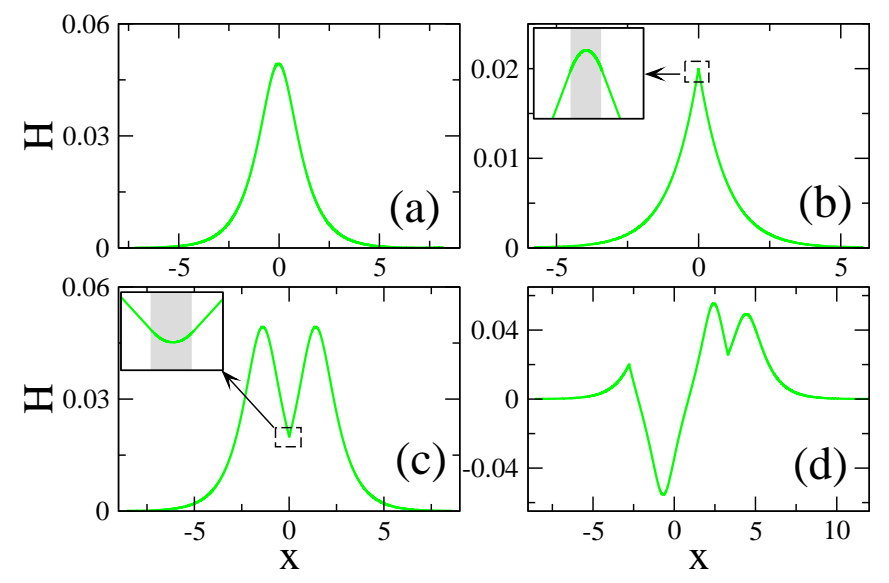

FIG. 2: (color online) Examples of different types of solitons: (a) fundamental soliton; (b,c) solitons with one domain of negative or positive magnetic permeability (shaded), respectively; (d) soliton with two different domains (shaded). Insets in $(b, c)$ show the magnified regions of the steep change of the magnetic field.

multi-stability, the type of the phase trajectories is defined by the corresponding branch of the multi-valued magnetic permeability. Correspondingly, different types of the spatial solitons appear when the phase trajectories correspond to the different branches of the nonlinear magnetic permeability.

The fundamental soliton is described by the separatrix trajectory on the plane $(H, d H / d x)$ that starts at the point $(0,0)$, goes around the center point $\left(0, H_{1}\right)$, and then returns back [see Fig. 1 solid curve]; the corresponding soliton profile is shown in Fig. 2(a). More complex solitons are formed when the magnetic permeability becomes multi-valued and is described by several branches. Then, soliton solutions are obtained by switching between the separatrix trajectories corresponding to different (upper and lower) branches of magnetic permeability. Continuity of the tangential components of the electric and magnetic fields at the boundaries of the domains with different values of magnetic permeability implies that both $H$ and $d H / d x$ should be continuous. As a result, the transitions between different phase trajectories should be continuous.

Figures 2(b,c) show several examples of the more complex solitons corresponding to a single jump to the lower branch of $\mu(H)$ (dotted) and to the upper branch of $\mu(H)$ (dashed), respectively. The insets show the magnified domains of a steep change of the magnetic field. Both the magnetic field and its derivative, proportional to the tangential component of the electric field, are continuous. The shaded areas show the effective domains where the value of magnetic permeability changes. Figure 2(d) shows an example of more complicated multi-hump soliton which includes two domains of the effective magnetic permeability, one described by the lower branch, and the other one - by the upper branch. In a similar way, we can find more complicated solitons with different number 


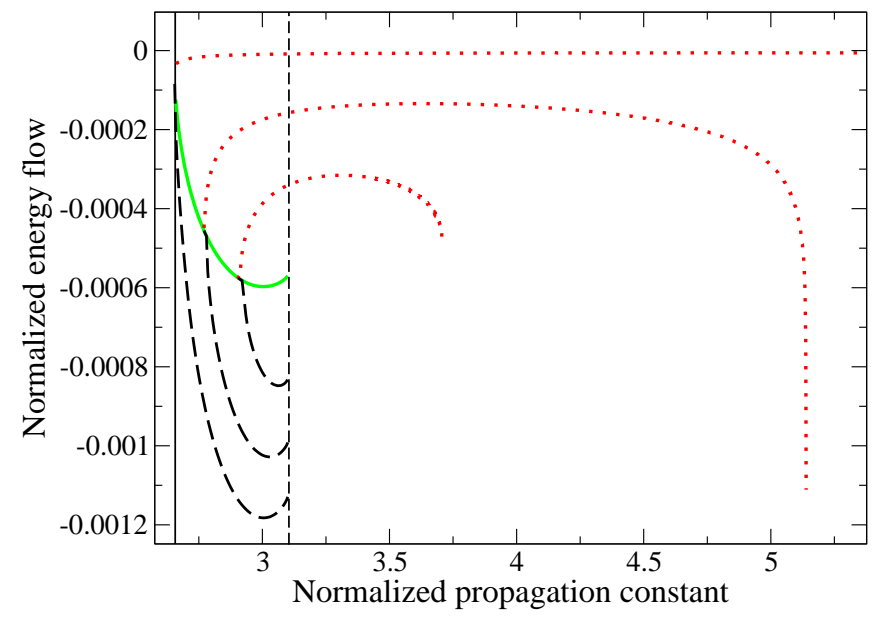

FIG. 3: (color online) Normalized soliton energy flow vs. its propagation constant. Vertical solid line is a boundary of wave localization in the linear limit, $\gamma=\gamma_{\mathrm{tr}}$. Thin vertical dashed line shows the existence boundary for the fundamental soliton. Thick solid curve shows the dispersion of a fundamental soliton. Dashed and dotted curves correspond to the solitons with one domain where the value of magnetic permeability changes. Dotted curves correspond to one-hump solitons shown in Fig. 2] (b), dashed curves correspond to the two-hump solitons shown in Fig. 2](c).

of domains of the effective magnetic permeability.

We note that some of the phase trajectories have discontinuity of the derivative at $H=0$ caused by infinite values of the magnetic permeability at the corresponding branch of $\mu_{\mathrm{eff}}(H)$. Such a non-physical effect is an artifact of the lossless model of a left-handed nonlinear composite considered here for the analysis of the soliton solutions. In more realistic models that include losses, the region of multi-stability does not extend to the point $H=0$, and in this limit the magnetic permeability remains a single-valued function of the magnetic field [6].

For such a multi-valued nonlinear magnetic response, the domains with different values of the magnetic permeability "excited" by the spatial soliton can be viewed as effective induced left-handed waveguides which make possible the existence of single- and multi-hump soliton structures. Due to the existence of such domains, the solitons can be not only symmetric, but also antisymmetric and even asymmetric. Formally, the size of an effective domain can be much smaller than the wavelength and, therefore, there exists an applicability limit for the obtained results to describe nonlinear waves in realistic composite structures.

Nonlinear dispersion of several types of spatial solitons is shown in Fig. 3 as the dependence of the soliton energy flow vs. the normalized propagation constant $\gamma$. The fundamental soliton exists in a limited range of $\gamma$. The lower boundary is determined by the threshold value $\gamma_{\mathrm{tr}}$. At the upper boundary, the amplitude of the soliton field exceeds the critical value $H^{*}$, and the magnetic permeability becomes positive. All spatial solitons are backward

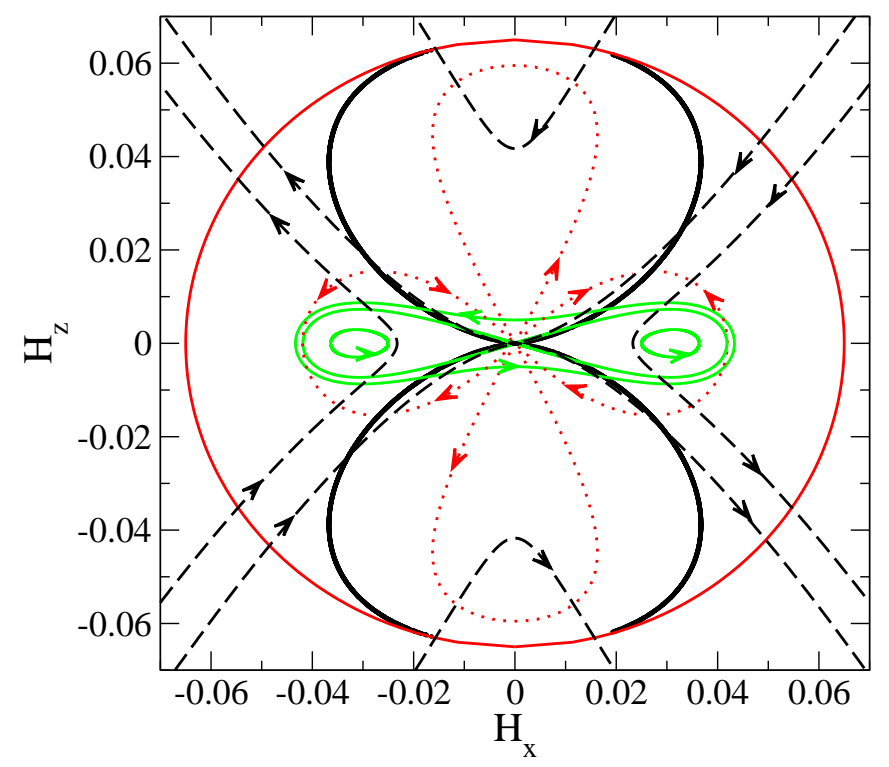

FIG. 4: (color online) Phase diagram of Eq. (6) corresponding to the multi-valued magnetic response shown in Fig. 1(inset). Solid, dashed and dotted curves mark the phase trajectories that correspond to solid, dashed and dotted curves in Fig. 1(inset), respectively. Inside the circle with the radius $H^{*}$ the magnetic permeability is a three-valued function of $H$, but it is single-valued, otherwise. Thick solid figure-eight curve is defined by a singularity in (6), at which the (dotted) phase trajectories change their direction.

propagating waves here, since the energy propagates in the direction opposite to the wavevector, as indicated by the negative sign of power flow.

When the infilling dielectric of the structure displays self-focusing nonlinear response, we have $\Omega<1$, and in such system we can find dark solitons, i.e. localized dips on the finite-amplitude background wave 7]. Similar to bright solitons, there exist both fundamental dark solitons and dark solitons with domains of different values of magnetic permeability. For self-defocusing nonlinearity and $\Omega<1$, magnetic permeability is a single-valued function, and such a nonlinear response can support dark solitons as well, whereas for self-focusing dielectric, and $\Omega>1$, no solitons can exist. Thus, in the composite with self-defocusing dielectric, depending on the frequency of the electromagnetic field, either bright or dark solitons can exist.

TE-polarized solitons. The TE-polarized waves are described by one component of the electric field and two components of the magnetic field. For such waves, magnetic permeability depends on two components of the magnetic field and the analysis of localized modes becomes more involved.

We look for the waves propagating in the $z$-direction,

$$
\mathbf{E}=\mathbf{y}_{\mathbf{0}} E e^{i \omega t-i h z}, \quad \mathbf{H}=\left(\mathbf{x}_{\mathbf{0}} H_{x}+\mathbf{z}_{\mathbf{0}} i H_{z}\right) e^{i \omega t-i h z},
$$

where $\left(\mathbf{x}_{\mathbf{0}}, \mathbf{y}_{\mathbf{0}}, \mathbf{z}_{\mathbf{0}}\right)$ are the unit vectors, and $E, H_{x}$, and $H_{z}$ are scalar real amplitudes of the fields. A constant 


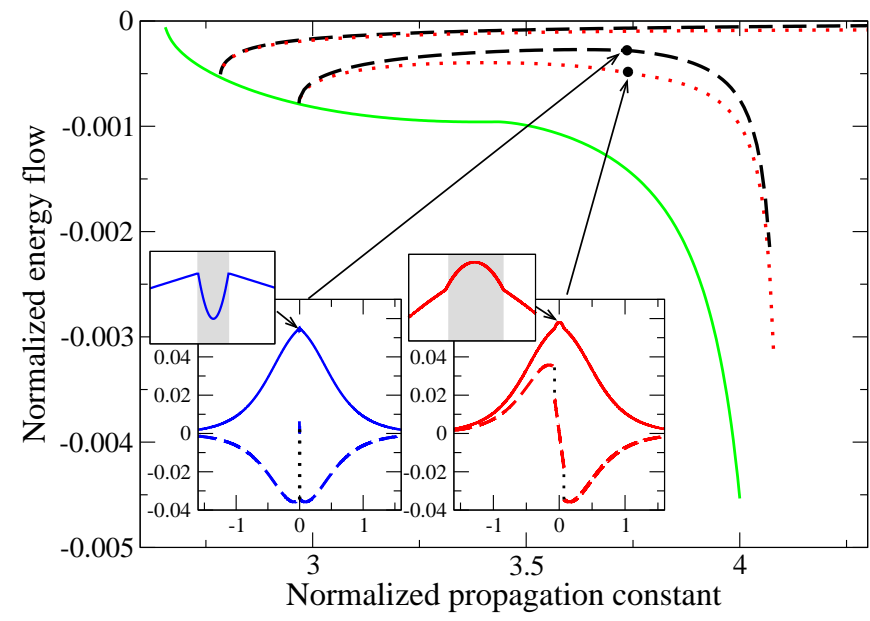

FIG. 5: (color online) Energy flow of the fundamental soliton (solid) and two solitons with a single domain (dashed and dotted). Solitons characterized by the dashed curves (left inset) have a single domain (shaded) with magnetic permeability described by the dashed curve in Fig. 11(inset). Solitons characterized by the dotted curves (right inset) have a domain (shaded) with magnetic permeability described by the dotted curve in Fig. 1(a, inset). Examples show the profiles of the components $H_{x}$ (solid) and $H_{z}$ (dashed) in the TE soliton.

phase difference between the components $E$ and $H_{z}$ is assumed, and no energy flow in the $x$-direction occurs. Spatial distribution of the magnetic field in a soliton is described by a system of coupled equations,

$$
\begin{array}{r}
\frac{d H_{z}}{d x}=\left[\epsilon \mu\left(H^{2}\right)-\gamma^{2}\right] H_{x} / \gamma \\
\frac{d H_{x}}{d x}=-\frac{\gamma^{2} \mu\left(H^{2}\right)+\xi\left[\epsilon \mu\left(H^{2}\right)-\gamma^{2}\right]}{\left[\gamma \mu\left(H^{2}\right)+\gamma \xi\right]}
\end{array}
$$

where $\xi=2 H_{x}^{2} \mu^{\prime}\left(H^{2}\right), H^{2}=H_{x}^{2}+H_{z}^{2}$. We analyze Eqs. (6) on the plane $\left(H_{x}, H_{z}\right)$, as shown in Fig. 4

Similar to the TM-polarized waves, the phase trajectory on the plane $\left(H_{x}, H_{z}\right)$ is defined by a particular branch of the multi-valued nonlinear magnetic permeability. Near the origin $(0,0)$, the phase trajectory is defined by the solid branch of the magnetic permeability [see Fig. 1(inset)]. In general, the phase diagram has three equilibrium states, which are similar to those described above for the TM-polarized waves. A singularity curve (thick solid line) is defined by the vanishing denominator in the second equation of Eqs. (6). The phase trajectories represented by dotted curves change their direction when they cross the singularity line.

For a homogeneous structure with the nonlinear magnetic permeability shown by a solid curve in Fig.11(inset), the separatrix curve on the phase plane of Fig. 4 describes the fundamental two-component TE-polarized spatial solitons with a smooth envelope. However, there exist more complex TE-polarized solitons, and such solitons include effective domains with different values of the multi-valued magnetic permeability. At the boundaries of the domains, both the fields $H_{x}$ and $H_{z} / \mu$ should be continuous. As a result, the phase trajectories describing the TE-polarized solitons with such domains will be discontinuous on the plane $\left(H_{x}, H_{z}\right)$. In Fig. 51 we show the energy flow for the family of the fundamental solitons and two types of solitons with a single domain, as the functions of the normalized propagation constant $\gamma$. Example of the solitons presented here correspond to a symmetric dependence of the $x$-component of the magnetic field; however, both antisymmetric and asymmetric TE-polarized solitons can exist in the structure as well.

In conclusion, we have analyzed both TE- and TMpolarized self-trapped nonlinear localized beams - spatial electromagnetic solitons - in left-handed metamaterials with nonlinear resonant response. We have revealed the existence of unique types of solitons supported by the hysteresis-type magnetic nonlinearity and the domains with different values of magnetic permeability. Such solitons can have symmetric, antisymmetric, or asymmetric structure. We believe that similar solitons can be found in other types of complex nonlinear materials with lefthanded properties or the frequency-dependent domains corresponding to negative refraction.

We thank Alex Zharov for useful discussions and acknowledge a support of the Australian Research Council.
[1] V. G. Veselago, Sov. Phys. Usp. 10, 509 (1968) [Usp. Fiz. Nauk 92, 517 (1967) (in Russian)].

[2] R.A. Shelby, D.R. Smith, and S. Schultz, Science 292, 77 (2001)

[3] C.G. Parazzoli, R.B. Greegor, K. Li, B.E.C. Koltenbah, and M. Tanielian, Phys. Rev. Lett. 90, 107401 (2003),

[4] A. Grbic and G.V. Eleftheriades, Phys. Rev. Lett. 92, 117403 (2004).

[5] T.J. Yen, W.J. Padilla, N. Fang, D.C. Vier, D.R. Smith, J.B. Pendry, D.N. Basov, and X. Zhang, Science 303, 1494 (2004).

[6] A.A. Zharov, I.V. Shadrivov, and Yu.S. Kivshar, Phys.
Rev. Lett. 91, 037401 (2003).

[7] For a comprehensive overview of spatial solitons in nonlinear optics, see Yu.S. Kivshar and G.P. Agrawal, Optical Solitons: From Fibers to Photonic Crystals (Academic Press, San Diego, 2003), 560 pp.

[8] V. B. Gil'denburg, A. V. Kochetov, A. G. Litvak, and A. M. Feigin, Sov. Phys. JETP 57, 28 (1983) [Zh. Eksp. Teor. Fiz. 84, 48 (1983)].

[9] M. I. Rabinovich and D. I. Trubetskov, Oscillations and Waves in Linear and Nonlinear Systems (Kluwer, Dordrecht, 1989). 